\title{
L'accent en proto-français : arguments factuels et typologiques contre l'influence du francique
}

\author{
Roland Noske \\ Université Lille 3 \& CNRS UMR 6183 'STL' \\ roland.noske@univ-lille3.fr
}

\section{Introduction}

Le français est souvent considéré comme la langue romane la plus germanique. Selon les descriptions historiques du français, le francique, dialecte germanique occidental, a influencé le français dans les domaines de la phonologie, de la morphologie, de la syntaxe et le lexique. Un des points majeurs de l'influence phonologique du francique serait l'introduction d'un accent d'intensité (c.-à-d. une augmentation de l'amplitude du signal) là où le latin avait un accent mélodique (consistant en une élévation de la fréquence fondamentale).

L'intensification de l'énergie articulatoire utilisée lors de la production de syllabes accentuées a mené entre autres à une diminution de l'énergie émise lors de la production des voyelles non accentuées, résultant en une réduction vers la voyelle centrale schwa, voire en une réduction totale ou effacement (syncope ou apocope).

Dans les descriptions classiques de l'histoire du français on trouve entre autres les passages suivants :

Pope (1952) :

The Frankish system of accentuation was a strong expiratory one and it was in the intensifying of the weak Latin tonic stress that the Germanic speech-habits, and in particular the Frankish, exercised their strongest influence in pronunciation. Directly resultant were: $<\ldots>$ the reduction, or effacement of the unstressed vowels $<\ldots>$. (1952:15)

Egalement plus loin dans ce livre :

Under the influence of the intensified tonic (= expiratory) stress of the Gallo-Roman period, atonic vowels in every type were ordinarily either effaced or reduced to e $(=ə),<\ldots>$.

$(1952: 112)$

Dans un autre manuel on lit :

... c'est au cours des IIIe et IVe siècles que la prosodie devient accentuelle. Les Francs, au Ve siècle, lui communiquent un surcroît de vigueur. $<\ldots>$ [l'intensité de l'accent] tend, en syllabe ouverte, $<\ldots>$ à affaiblir les voyelles atones jusqu'à les faire disparaître.

(Zink $1986: 37$ )

Et dans un troisième :

... la voyelle tonique portait en germanique un accent bien plus fort qu'en latin vulgaire. Ces deux faits, (.....), et l'accentuation germanique, ont eu des résultats d'une importance considérable.

(Von Wartburg (1965:65)

Et le même auteur explique de la façon suivante la réduction de voyelle et la séparation de la langue d'oïl et la langue d'oc : 


\begin{abstract}
Dans la Gaule septentrionale les voyelles toniques dominent le mot par leur accent beaucoup plus que dans le Midi $\langle\ldots . .<$. $<\ldots>$ Si les voyelles toniques portent un accent très fort, elles absorbent une très grande partie de l'énergie articulatoire dispensée pour un mot. Il s'ensuit un affaiblissement des autres syllabes, non accentuées. $<\ldots .>$... les voyelles atones disparaissent ou ne se maintiennent qu'à peine et sous une forme amoindrie.

$(1965: 61)$
\end{abstract}

La Chaussée (1989: 193), dans une description des changements de la fin du Ve / début Ve siècle, attribue également la syncope (de voyelles post-toniques) à l'influence germanique.

A part la réduction de voyelle et l'effacement de voyelle, l'accent final qui caractérise le français moderne, est indirectement attribué à l'influence germanique, cf. Kukenheim (1971) :

Mais la prononciation des Germains, qui frappait à coups de marteau la syllabe accentuée et qui, par là, a provoqué la chute de la voyelle finale (non accentuée) au VIIe siècle, doit avoir créé des circonstances favorables à l'oxytonisme français.

(1971:319)

On constate donc que les auteurs cités attribuent à l'influence germanique une grande partie des changements majeurs phonologiques qu'a subis le français depuis sa genèse. Selon eux, le francique du Ve siècle se caractérisait par un "strong expiratory [stress]» (Pope), un "surcroît de vigueur» (Von Wartburg), ou même une accentuation « à coups de marteau » (Kukenheim). Cependant, ces affirmations ne s'appuient sur aucune référence à des recherches qui prouvent que le francique, dans cette période prélittéraire de la langue, aurait eu effectivement un fort accent d'intensité. De même, les descriptions de l'ancien néerlandais (bas-francique) que nous avons consultées ne contiennent aucune référence à un accent à grande intensité. Pour l'ancien haut-allemand, on trouve effectivement des affirmations de ce type dans les manuels tels que Braune (1975 : 13) qui parle d'un fort accent de mot expiratoire («stark expiratorisches Wortakzent ») dans l'évolution de l'accent libre indo-européen vers l'accent initial en proto-germanique. Cependant, cette idée ne paraît pas justifiée à la lumière de recherches typologiques récentes sur l'histoire du haut-allemand telles que celles de Nübling et Schrampke (2004) et de Szczepaniak (2007). Ces auteurs montrent que l'accent en ancien haut-allemand était de faible intensité et qu'il était surtout mélodique. Or, si l'on refuse de prendre pour acquise l'idée que l'accentuation forte en gallo-roman ait ses origines en ancien francique ${ }^{1}$ et que l'on applique certains tests de vérification, on arrive à des résultats et des paradoxes étonnants qui feront l'objet de la section suivante.

\title{
2 Arguments contre une influence francique en matière d'accentuation
}

Dans cette section, nous montrerons qu'il est extrêmement improbable que le gallo-roman ait emprunté son accent d'intensité lourd au francique et ce pour diverses raisons :

i. parce que les emprunts d'accentuation sont rares ou inexistants ;

ii. parce que le francique du Ve siècle avait probablement encore l'accent initial du germanique commun qui, s'il y avait eu transfert, aurait également été transféré ;

iii. et finalement, parce que, en vue de l'absence de réduction de voyelles réduites, le francique à cette époque-là n'avait pas un accent d'intensité lourd.

Regardons ces arguments plus en détail.

\subsection{Les systèmes d'accentuation ne sont pas transférés d'une langue à l'autre}

La littérature sur les langues en contact (par exemple Weinreich 1963, Appel et Muysken 1987, Thomason 2001, Winford 2002) ne mentionne pas d'exemples de transfert d'un système accentuel d'une langue à une autre. Le seul type de transfert accentuel est un tranfert lié à des mots empruntés ou des morphèmes empruntés. ${ }^{2}$ Le seul autre exemple connu en linguistique historique, hormis celui dont il est 
question ici, est celui de l'anglais, qui aurait emprunté son système d'accentuation actuel (accentuation trochaïque au côté droite, ne prenant pas en compte la dernière syllabe) au latin médiéval. Cependant, cette hypothèse a été réfutée récemment par Fournier (2007). Le transfert de l'accentuation du francique vers le gallo-roman serait donc très exceptionnel.

\subsection{La place de l'accent dans le francique du Ve siècle}

Hormis quelques mots dans la Loi Salique ${ }^{4}$ du VIe siècle, nous ne disposons d'aucune source écrite de cette dernière langue datant de l'époque du prétendu emprunt de l'accent d'intensité par le gallo-roman au francique. ${ }^{5}$ Cependant, nous savons que qu'autour du Ve siècle les dialectes germaniques accentuaient la première syllabe de la racine du mot. ${ }^{6}$ Ainsi, en néerlandais moderne, l'accentuation est sur le premier trochée de droite dans les mots morphologiquement simples (Booij 1995: 101). Or, si l'accentuation francique a vraiment exercé une telle influence sur le gallo-roman, on s'attendrait à ce que la place de l'accent aurait également été transférée. Donc, le gallo-roman devrait avoir alors un accent initial, ce qui n'est pas le cas.

\subsection{Le prétendu accent d'intensité fort en francique}

Pope (1952) et Zink (1986) attribuent explicitement l'accent d'intensité qui a provoqué la réduction de voyelle, ainsi que la syncope et l'apocope en gallo-roman, à un emprunt au francique. Or, la typologie des langues montre que la réduction des contrastes entre voyelles, partielle ${ }^{7}$ ou totale, ainsi que les processus d'apocope et de syncope, sont très courants dans les langues avec un accent d'intensité fort. On s'attendrait donc à ce qu'en germanique aussi la présumée accentuation "à coups de marteau », cause un processus de réduction de voyelle, ainsi que des processus d'effacement de voyelle.

Cependant, l'examen des caractéristiques de l'ancien haut allemand et de l'ancien néerlandais montre que ces langues n'avaient ni de processus de réduction de voyelles, ni de processus d'effacement de voyelle. La réduction de voyelle et divers processus de syncope et d'apocope ont bien eu lieu en haut allemand et en néerlandais, mais beaucoup plus tard, c.-à-d. à partir de la fin du XIe siècle. Dans la périodisation de ces langues, ces processus marquent précisément la transition de l'ancien haut allemand et de l'ancien néerlandais respectivement vers le moyen haut allemand et le moyen néerlandais (König 1978 :73, Quak $1997: 37)$.

Voici, en guise d'illustration, trois exemples de textes antérieurs à la fin du XIe siècle, l'un en haut francique, les deux autres en bas francique. Pour le haut francique, voici les deux premières lignes d'une version du Notre Père en ancien haut francique (plus précisément en francique rhénan méridional, le catéchisme de Wissembourg du IXe siècle, source : Braune 1994) :

(1) Fater unsēr thu in himilom bist giuuīhit sī namo thīn.

père notre toi dans cieux es sanctifié soit nom ton

Pour l'exemple en ancien haut francique on peut constater que, dans le mot himilom, 'ciel' (datif), où l'accent principal tombe sur la première syllabe, les voyelles des deux syllabes suivantes sont des voyelles pleines. De même, la deuxième voyelle de namo 'nom' (nominatif), où l'accent tombe également sur la première syllabe, n'a pas été réduite.

Comparons cet exemple aux deux premières lignes de la même prière en moyen haut allemand (autour de 1300) :

(2) vater unser der da bist in den himeln, geheiliget wert din name.

père notre qui là es dans les cieux sanctifié est ton nom

Ici, on voit (parmi d'autres changements, tels que l'apparition d'articles) qu'en ancien haut francique le deuxième $i$ de himilon s'est réduit à un $e$ ([ə]), tout comme le $o$ de namo. En outre, contrairement à ce qui se passe dans le texte en ancien haut francique, la troisième voyelle de himilom a été syncopée. ${ }^{8}$ Cela 
montre que les processus de réduction et d'effacement de voyelles ne sont devenus opérationnels qu'après le IXe siècle.

Regardons maintenant un texte en ancien bas francique oriental (limbourgeois), à savoir les Psaumes de Wachtendonck, qui datent du Xe siècle (Van Helten 1902, Cowan 1957, Kyes 1989).

(3) Forchta in biuonga quamon ouer mi in bethecoda mi thuisternussi Angoisses et tremblements venaient sur moi et couvrit moi obscurité

'Je fus pris par des angoisses et par des tremblements et de l'obscurité me couvrit'

En néerlandais moyen et moderne (à la base desquels se trouve principalement le bas francique), le $o$ dans quamon s'est réduit à [ə] (kwamen ['kua:mən] en néerlandais moderne).

Notre troisième exemple vient du bas francique occidental. Ce texte, trouvé dans une bibliothèque à Oxford aux années trente du siècle dernier, date du troisième quart du XIe siècle et a été écrit probablement par un moine flamand dans l'abbaye de Rochester. Ce texte a longtemps été considéré comme étant le texte néerlandais le plus ancien (cf. Schönfeld 1933).

(4) Hebban olla vogala nestas hagunnan hinase hic enda thu uuat unbidan uue nu ont tous oiseaux nids commencé sauf moi et toi que attendons nous maintenant

'Tous les oiseaux ont commencé des nids, sauf moi et toi. Qu'attendons-nous alors ?'

Si l'on compare cette phrase avec son équivalent en néerlandais moyen et moderne, on constate que beaucoup de voyelles ont été remplacées pas $e$. Ainsi, hebban correspond à hebben [hebən] en néerlandais moyen, vogala à vogele, nestas à nesten, et le suffixe de l'infinitif de unbidan, -an, à -en.

Nous voyons donc qu'en francique, la réduction de voyelle, ainsi que les processus de syncope et d'apocope n'ont pas eu lieu avant la fin du XIe siècle, contrairement à ce qui s'est passé en gallo-roman. Effectivement, l'étude la plus approfondie sur la périodisation de la réduction de voyelle en français, celle de Richter (1934), note que la réduction de voyelles et la syncope et l'apocope ont eu lieu entre la fin du IVe siècle et la fin du VIe siècle $(1934: 202){ }^{9}$

Or, il serait étonnant si le prétendu « surcroît de vigueur » qu'aurait eu le germanique autour du Ve siècle et qui serait responsable de la réduction de voyelle et les phénomènes de syncope et apocope en galloroman, n'aurait pas eu le même effet en ancien francique. ${ }^{10}$

\section{Autres évolutions en français et en francique}

Les arguments avancés dans la section précédente suffisent à eux seuls pour réfuter les affirmations des manuels concernant l'influence accentuelle du francique sur le gallo-roman. Ils mènent en effet à la conclusion que le mouvement vers un accent d'intensité fort a été un mouvement autonome du galloroman. ${ }^{11}$ Nous montrerons dans la section 4 que ce changement a été un élément dans l'évolution typologique plus générale qui a eu lieu en gallo-roman. Pour comprendre cette évolution typologique, il faut d'abord relever quelques changements autres que la réduction et l'effacement de voyelle qui ont eu lieu dans l'histoire du français, ainsi que quelques changements dans l'histoire du germanique occidental.

\subsection{L'évolution du français en termes de complexité syllabique}

Comme on l'a souvent signalé (cf. Jacobs 1992), il y a eu un mouvement pendulaire dans la structure syllabique dans l'évolution du latin classique au français moderne. Les étapes de l'évolution du latin classique vers le latin vulgaire tardif se caractérisent de la façon suivante :

(i) du latin classique vers le latin vulgaire tardif: par un mouvement vers une structure syllabique moins compliquée (en raison de plusieurs processus d'épenthèse) ;

(ii) du latin vulgaire tardif vers le gallo-roman et ensuite vers l'ancien français précoce : par une structure syllabique plus complexe ; 
(iii) de l'ancien français précoce vers l'ancien français tardif, le moyen français et le français moderne : par un mouvement progressif vers une structure syllabique beaucoup plus ouverte.

Le mouvement peut donc être schématisé ainsi : fermé $>$ ouvert $>$ fermé $>$ ouvert.

\subsection{Autres aspects de l'évolution typologique du français}

L'évolution en termes de structure syllabique ne représente qu'une partie de l'évolution typologique du français, à côté de la réduction de voyelle et les processus d'effacement de voyelle (syncope, apocope) commentés ci-dessus. Nous relevons d'autres points dans ce qui suit. Ces évolutions joueront un rôle dans la partie qui sera consacrée à la typologie.

\subsubsection{Diphtongaison}

Il y a eu des diphtongaisons du IIIe jusqu'au VIe siècles, sous l'influence dans des syllabes accentuées

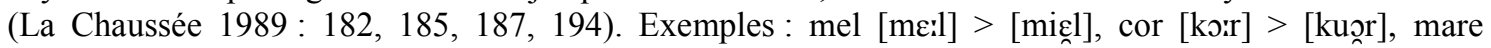
['ma:re] > ['mą̧rə].

\subsubsection{Dégémination}

Les consonnes géminées du latin tardif et celles produites par l'assimilation dal la période gallo-romane étaient réduites à des consonnes simples en ancien français. Cela s'est passé à partir du $9 \mathrm{e}$ siècle (Pope 1952:147, Bourciez 1930:305).

\subsubsection{Genèse du dévoisement final}

Comme nous l'avons vu déjà plus haut, le stade (ii) se caractérise par un renforcement de l'accent de l'intensité, d'une réduction de voyelles non accentuées et de différents processus d'effacement des voyelles non accentuées. En outre, il y a eu, à partir du VIIIe siècle, un processus de dévoisement d'obstruantes finales (Pope 1952 : 98, avec les exemples translittérés en API) :

(5) a. [lungum] $>$ [lunk]

b. [grandem] > [grãnt]

c. $[$ perdo] $>$ [pert]

d. [risum] $>*$ [rizu $]>[$ ris $]$

e. $[$ serwum $]>*[$ serva $]>[$ serf $]$

Pope remarque que ce processus a eu lieu dans une période où l'accent d'intensité était fort. Dans la typologie des langues (voir la discussion sur les langues de mot et les langues syllabiques ci-dessous dans la section 4), les langues de mots ont plus tendance à avoir des signaux démarcatifs que les langues de syllabes.

\subsubsection{Perte de productivité de la réduction de voyelle}

Dans le stade (iii), le mouvement vers la période du moyen français et du français moderne, la réduction de voyelle a perdu sa productivité : les voyelles inaccentuées nouvellement introduites dans la langue, par exemple par des emprunts, ne sont plus réduites au schwa.

\subsubsection{Perte de productivité du dévoisement final}

De même, mis à part quelques vestiges (tels que les alternances lexicalisées en neuf $\sim$ neuve et le [t] dans grand ami [grãtami]), le dévoisement final a disparu. Plus bas, nous montrerons en plus que la simultanéité de la perte de productivité de ces deux processus n'est pas due au hasard: elle est la conséquence d'un mouvement typologique unique. 


\subsubsection{Changement de conditionnement de l'effacement de voyelle}

L'effacement de voyelle (du schwa), quant à lui, a continué à exister en moyen français et en français moderne, mais il a assumé un tout autre rôle : au lieu d'être conditionné par un accent d'intensité, une grande partie de sa raison d'être est désormais une optimation de la structure syllabique, comme l'effacement du schwa en position prévocalique : ${ }^{12}$

(6) le + homme > l'homme

Nous montrerons que ce changement de conditionnement de l'effacement du schwa est également la conséquence de l'évolution typologique du français.

\subsection{Quelques aspects de l'évolution du germanique occidental continental}

Tout comme le français, le francique et les autres dialectes du germanique occidental ont subi de grands changements depuis la période des premières sources écrites jusqu'à nos jours. Les évolutions qui nous concernent sont :

- la genèse, déjà mentionnée en section 2.3 , du processus de réduction de voyelle dans la transition de l'ancien haut allemand et de l'ancien néerlandais vers le moyen haut allemand et le moyen néerlandais ;

- le développement de processus de syncope et d'apocope en moyen haut allemand ${ }^{13}$ et en moyen néerlandais ;

- la genèse en période du moyen haut allemand, et déjà partiellement en ancien néerlandais ${ }^{14}, \mathrm{du}$ processus de dévoisement final ;

- L'évolution d'un système d'accentuation initial en germanique commun, non sensible à la quantité, vers un système accentuel où la quantité syllabique joue un rôle.

\section{Les langues comptant les syllabes et les langues comptant les mots}

Pour mettre en perspective les évolutions du français et du francique, il s'impose un détour par la théorie sur la typologie des langues de syllabes et les langues de mots, telle que proposée par Auer (1993, 1994, 2001), Auer \& Uhmann (1988). Pour expliquer cette théorie, il me faut revenir un peu dans le passé et mentionner brièvement trois développements antérieurs, sur lesquels Auer et Uhmann se basent.

\subsection{Isochronie syllabique vs. isochronie accentuelle}

Une typologie phonétique des langues à isochronie syllabique vs. celles à isochronie d'accent a été proposée par Pike (1945) et par Abercrombie (1967). Selon cette typologie, une langue comme le français (contemporain) est une langue à isochronie syllabique, c.-à-d. que dans une telle langue, les distances temporelles entre toutes les syllabes sont identiques. Par contre, dans une langue à isochronie accentuelle, comme l'anglais, c'est les distances temporelles entre les syllabes accentueés qui sont identiques.

Cette typologie a été critiquée par plusieurs auteurs, entre autres par Wenk \& Wioland (1982) et par Roach (1982), simplement parce que les recherches relevant de la phonétique expérimentale la contredisent.

\subsection{La théorie perceptuelle de Dauer}

Cependant, cette typologie a été revitalisée par Dauer 1983, 1987), qui se base sur des critères perceptuels. Dauer (1983) constate qu'une langue comme l'anglais, où les intervalles entre les syllabes accentuées consistent de 5 syllabes au maximum, fait preuve d'une plus grande stabilité en ce qui concerne la longueur de pieds qu'une langue telle que l'espagnol, l'italien, et le grec (et l'on pourrait y 
ajouter le français), où ces intervalles peuvent aller jusqu'à 10 syllabes. Tenant compte de ces faits, elle émet l'hypothèse suivante :

\author{
I would like to propose that the rhythmic differences we feel to exist between \\ languages such as English and Spanish are more a result of phonological, phonetic \\ lexical and syntactic facts about that language than any attempt on the part of the \\ speaker to equalize interstress or intersyllabic intervals \\ (Dauer 1983 : 55, également citée dans Szczepaniak $2007: 20$ )
}

Elle constate que les langues qui sont perçues comme ayant une isochronie syllabique, ont en général une structure syllabique simple avec beaucoup de syllabes ouvertes et sans corrélation entre accent et poids syllabique. Par contre, les langues qui sont perçues comme ayant une isochronie accentuelle, ont une structure syllabique complexe, une avec beaucoup de syllabes fermées, et avec une tendance à l'accentuation des syllabes fermées. En outre, dans les langues perçues comme ayant une isochronie syllabique, les possibilités de contraste qualitatif entre les voyelles accentuées et celles entre les voyelles non-accentuées sont identiques, à l'opposé des langues perçues comme ayant une isochronie accentuelle, où il y a réduction de voyelle phonétique ou phonologique. En ce qui concerne l'accentuation, les langues perçues comme ayant une isochronie syllabique ont un accent de mot phonétiquement faible ou tout à fait absent, tandis que les langues perçues comme ayant une isochronie accentuelle ont un accent de mot bien perceptible et, en outre, des règles grammaticales qui réfèrent à l'accent.

Dans sa publication de 1987, Dauer ajoute qu'en ce qui concerne les consonnes, on trouve dans des langues perçues comme ayant une isochronie accentuelle des allophones déterminées par l'accent et que l'accent dans ces langues est souvent libre. En ce qui concerne les voyelles, elle constate que toujours dans les langues perçues comme ayant une isochronie accentuelle, les syllabes accentuées sont en général plus longues que les syllabes inaccentuées.

\title{
4.3 La phonologie 'prosodique' de Nespor et Vogel
}

Il faut également mentionner ici la théorie de la phonologie dite 'prosodique"15, telle que proposée par Nespor et Vogel (1986). Nespor et Vogel proposent un système de constituantes phonologiques organisé de façon hiérarchique. Dans ce système, une ou plusieurs constituantes d'une catégorie sont licenciées par la constituante qui appartient à la catégorie immédiatement supérieure, ce qui implique qu'une constituante ne peut appartenir à deux constituantes supérieures à la fois. Les catégories de constituantes sont : la syllabe phonologique $(\sigma)$, le pied phonologique $(F)$, le mot phonologique $(\omega)$, le groupe clitique $(\mathrm{C})$, la 'phrase' phonologique ( $\varphi$, 'phonological phrase'), la constituante intonationelle (I), l'énoncé phonologique (U).

\subsection{La théorie d'Auer, Uhmann et Szczepaniak : langues de syllabes et langues de mots}

Dans ce traitement de la typologie phonólogique, nous en arrivons maintenant à notre but principal, c.-à.-d. la théorie d'Auer (1993, 1994, 2001), d'Auer \& Uhmann (1988) et de Szcepaniak (2007). Ces auteurs, proposent, en se basant partiellement sur les idées de Bauer (voir la section 4.2), ainsi que sur celles de Nespor et Vogel (voir 4.3), une typologie multifactorielle scalaire dont les extrêmes sont les langues comptant les syllabes (ou simplement langues syllabiques) et les langues comptant les accents, ou langues de mots, ('word language', 'Wortsprache'. Dans les langues syllabiques, la constituante principale est la syllabe, tandis que dans les langues de mots c'est le mot phonologique. Ces deux types de langue sont, bien sûr, des prototypes.

Le terme de constituante principale signifie : la catégorie à laquelle se réfèrent la plupart des règles distributionnelles, ainsi que la plupart des processus phonétiques et phonologiques. Ainsi, en ce qui concerne les règles distributionnelles par exemple, dans une langue syllabique, les frontières entre les syllabes sont nettes, tandis qu'elles ne sont pas claires dans une langue de mots. La structure de mots ne joue pas de rôle dans l'accentuation des langues syllabiques, tandis qu'elle importe beaucoup dans les 
langues de mots. Pour une aperçue détaillée des différences entre les langues de syllabes et les langues de mots, ainsi qu'une explication détaillée de la théorie, voir Szczepaniak (2007 : 7-56).

Les éléments concrets qui sont le plus importants ici, sont donnés dans le tableau $1^{16}$

tableau 1: critères pour déterminer le statut d'une langue sur l'échelle langues syllabique - langue de mots (schéma adapté de de Nübling \& Schrambke $2004: 284-285)^{17}$

\begin{tabular}{|c|c|c|c|}
\hline $\mathrm{n}^{\mathrm{o}}$ & critère & $\begin{array}{l}\text { langues syllabiques } \\
\rightarrow \text { comptant les syllabes } \\
\text { syllabe comme unité } \\
\text { prosodique de base (longueur } \\
\text { de pied variable) }\end{array}$ & $\begin{array}{l}\text { langues de mots } \\
\rightarrow \text { comptant les accents } \\
\text { mot phonologique comme unité } \\
\text { prosodique de base (longueur de } \\
\text { syllabe variable) }\end{array}$ \\
\hline 1 & structure syllabique & $\begin{array}{l}\text { syllabes CV (rarement des } \\
\text { syllabes fermées); } \\
\text { toutes les syllabes de longueur } \\
\text { égale. }\end{array}$ & $\begin{array}{l}\text { types de syllabes variables, de } \\
\text { complexité différente, en fonction de la } \\
\text { position de l'accent; différences } \\
\text { fréquentes entre les syllabes médiales } \\
\text { et périphériques. }\end{array}$ \\
\hline 2 & frontières syllabiques & $\begin{array}{l}\text { frontières de syllabe bien } \\
\text { reconnaissables et constantes }\end{array}$ & $\begin{array}{l}\text { frontières de syllabe mal } \\
\text { reconnaissables, variables et dépendant } \\
\text { du débit de la parole }\end{array}$ \\
\hline 3 & géminées & géminées possibles & $\begin{array}{l}\text { réduction de géminées, sauf à des } \\
\text { endroits où elles sont pertinentes du } \\
\text { point de vue morphologique, par ex. } \\
\text { dans des frontière internes de mots } \\
\text { composés, par ex. all. Schifffahrt [f:] (= } \\
\text { un [f] long). }\end{array}$ \\
\hline 4 & effets d'accentuation & $\begin{array}{l}\text { pas / peu de différences en } \\
\text { structure entre syllabes } \\
\text { accentuées et inaccentuées }\end{array}$ & $\begin{array}{l}\text { syllabes accentuées = syllabes lourdes; } \\
\text { syllabes inaccentuées = syllabes } \\
\text { légères ; diphtongaisons }\end{array}$ \\
\hline 5 & $\begin{array}{l}\text { assignation de } \\
\text { l'accent }\end{array}$ & $\begin{array}{l}\text { surtout basée sur la syllabe, } \\
\text { absence possible d'accent de } \\
\text { mot fixe. }\end{array}$ & $\begin{array}{l}\text { règles d'accentuation (souvent com- } \\
\text { plexes) déterminées par la morphologie } \\
\text { / le lexique / la sémantique }\end{array}$ \\
\hline 6 & phonotactique & $\begin{array}{l}\text { phonotactique régulière et } \\
\text { stable, pas d'allophones } \\
\text { déterminées par leur position }\end{array}$ & $\begin{array}{l}\text { signaux de démarcation de mots, allo- } \\
\text { phones déterminés par la position (ini- } \\
\text { tiale, médiale, finale ...), des } \\
\text { restrictions phonotactiques }\end{array}$ \\
\hline 7 & vocalisme & $\begin{array}{l}\text { peu de différences entre } \\
\text { voyelles accentuées et } \\
\text { inaccentuées; degré de } \\
\text { tension relativement égal. }\end{array}$ & $\begin{array}{l}\text { beaucoup de différences entre voyelles } \\
\text { accentuées et inaccentuées (allemand, } \\
\text { danois, anglais) ; voyelles accentuées : } \\
\text { souvent des distinctions de longueur ; } \\
\text { voyelles inaccentueés : centralisations } \\
\text { (réductions) }\end{array}$ \\
\hline 8 & $\begin{array}{l}\text { harmonie vocalique, } \\
\text { métaphonie }\end{array}$ & possible & rare \\
\hline 9 & effacement de voyelle & $\begin{array}{l}\text { pour des raisons d'optimation } \\
\text { de la structure syllabique }\end{array}$ & pour des raisons accentuelles \\
\hline 10 & liaison & $\begin{array}{l}\text { oui (à travers des frontières de } \\
\text { morphème) }\end{array}$ & $\begin{array}{l}\text { non (signaux de démarcation, par ex. } \\
\text { coup de glotte) }\end{array}$ \\
\hline
\end{tabular}

Evidemment, une langue de mots et une langue syllabique représentent des types idéaux, et en réalité chaque langue se place quelque part entre les deux extrémités. De même, tous les critères ne sont pas pertinents pour chaque langue. Les idées avancées par Auer et Uhmann ont jusqu'ici reçu (trop) peu d'attention, peut-être parce la plupart des publications sur ce sujet ont été rédigées en allemand. 
Si nous examinons où se trouve le français moderne sur cette échelle, il ressort, selon les symptômes possibles relevés plus haut que, qu'il s'agit d'une langue syllabique. Selon huit de ces critères au moins, le français moderne est en effet une langue syllabique :

tableau 2 : critères du tableau 1 qui déterminent que le français moderne est une langue syllabique

\begin{tabular}{|l|l|l|}
\hline $\mathrm{n}^{\mathbf{0}}$ & critère & raison \\
\hline 1 & structure syllabique & beaucoup de syllabes ouvertes (voir section 3.1) \\
\hline 2 & frontières syllabiques & $\begin{array}{l}\text { la position des frontières de syllabe ne sont pas équivoques comme en } \\
\text { allemand et en anglais (cf. les consonnes 'ambisyllabiques' dans ces } \\
\text { langues), mais claires. }\end{array}$ \\
\hline 4 & effets d'accentuation & $\begin{array}{l}\text { les syllabes inaccentuées ne sont pas phonologiquement plus longues que } \\
\text { les syllabes inaccentuées }\end{array}$ \\
\hline 5 & $\begin{array}{l}\text { assignation de } \\
\text { l'accent }\end{array}$ & $\begin{array}{l}\text { l'accentuation est basée sur la syllabe, non pas sur la morphologie, etc., } \\
\text { pas de paires minimales de mots qui ne se distinguent que par la place de } \\
\text { l'accent, comme dans les langues germaniques }\end{array}$ \\
\hline 6 & phonotactique & $\begin{array}{l}\text { pas d'allophones déterminés pas leur position, par ex., pas de voisement } \\
\text { intervocalique, pas de dévoisement final (voir section 3.2.4), pas d'aspi- } \\
\text { ration initiale }\end{array}$ \\
\hline 7 & vocalisme & $\begin{array}{l}\text { peu de divergence entre des voyelles accentuées et inaccentuées, pas de } \\
\text { réduction de réduction de voyelle synchronique (voir section 3.2.3) }\end{array}$ \\
\hline 9 & $\begin{array}{l}\text { effacement de } \\
\text { voyelles }\end{array}$ & $\begin{array}{l}\text { effacement de voyelles pour des raisons d'optimation de syllabe (voir } \\
\text { section 3.2.5) }\end{array}$ \\
\hline 10 & liaison & $\begin{array}{l}\text { existence de liaison pour des raisons d'optimation de la structure } \\
\text { syllabique }\end{array}$ \\
\hline
\end{tabular}

Si l'on applique les critères du tableau 1 à l'ancien français, on voit qu'il y a au moins six critères qui militent en faveur d'une catégorisation comme langue de mots :

tableau 3 : critères du tableau 1 qui déterminent que le gallo-roman et l'ancien français sont des langues de mots

\begin{tabular}{|l|l|l|}
\hline $\mathrm{n}^{\mathrm{o}}$ & critère & raison \\
\hline 1 & structure syllabique & $\begin{array}{l}\text { beaucoup de syllabes complexes, grande variabilité entre types de syllabe } \\
\text { (voir section 3.1) }\end{array}$ \\
\hline 3 & géminées & réduction de géminées (voir section 3.2.2) \\
\hline 4 & effets d'accentuation & diphtongaisons du III au VIe siècles (voir section 3.2.2) \\
\hline 6 & phonotactique & $\begin{array}{l}\text { dévoisement final comme signal de démarcation de mots (voir section } \\
3.2 .1 \text { ) }\end{array}$ \\
\hline 7 & vocalisme & réduction de voyelles inaccentuées à schwa \\
\hline 9 & effacement de voyelle & $\begin{array}{l}\text { effacement de voyelles (syncope, apocope) sous l'influence de l'accent } \\
\text { (voir section 3.2.5) }\end{array}$ \\
\hline
\end{tabular}

Nous pouvons constater par ces critères qu'entre la période de l'ancien français et celle du français moderne, la langue s'est transformée d'une langue de mots à une langue syllabique.

Examinons maintenant le haut allemand et le néerlandais avec les mêmes critères. D'abord nous regardons le haut allemand moderne et le néerlandais moderne, cf. tableau 4 : 
tableau 4 : critères du tableau 1 qui déterminent que le haut allemand moderne et le néerlandais moderne sont des langues de mots

\begin{tabular}{|c|c|c|}
\hline $\mathrm{n}^{\mathrm{o}}$ & critère & raison \\
\hline 1 & structure syllabique & $\begin{array}{l}\text { complexes, types de syllabe variables, en fonction de l'accent et de la } \\
\text { morphologie }\end{array}$ \\
\hline 2 & frontières syllabiques & $\begin{array}{l}\text { frontières de syllabes frontières de syllabe mal reconnaissables, } \\
\text { ambisyllabicité }\end{array}$ \\
\hline 3 & géminées & $\begin{array}{l}\text { réduction de géminées à partir de la période de moyen haut allemand et du } \\
\text { moyen néerlandais }\end{array}$ \\
\hline 4 & effets d'accentuation & $\begin{array}{l}\text { aspiration de plosive initiales en nouveau haut-allemand, diphtongaison } \\
\text { phonétiques de voyelles accentuée en néerlandais modernen s }\end{array}$ \\
\hline 5 & $\begin{array}{l}\text { assignation de } \\
\text { l'accent }\end{array}$ & $\begin{array}{l}\text { règles accentuation complexes, dépendant de la morphologie, préférence à } \\
\text { accentuer les syllabes lourdes }\end{array}$ \\
\hline 6 & phonotactique & signaux démarcatifs : insertion de coup de glotte, dévoisement final \\
\hline 7 & vocalisme & réduction de voyelle productive en néerlandais moderne \\
\hline 8 & $\begin{array}{l}\text { harmonie vocalique, } \\
\text { métaphonie }\end{array}$ & $\begin{array}{l}\text { la métaphonie phonologique n'est plus phonologiquement productive en } \\
\text { nouveau haut allemand, s'est morphologisée }\end{array}$ \\
\hline 9 & $\begin{array}{l}\text { effacement de } \\
\text { voyelles }\end{array}$ & $\begin{array}{l}\text { la syncope et l'apocoe sont conditionées par l'accent, comme dans all. ich } \\
\text { hab' (pour ich habe), néerl. vreeslijk (pour vreselijk) }\end{array}$ \\
\hline 10 & liaison & en général inexistant, au lieu de cela : insertion de coup de glotte \\
\hline
\end{tabular}

Par contre, l'ancien haut allemand et l'ancien néerlandais étaient placés beaucoup plus vers les langues syllabiques sur l'échelle :

tableau 5 : critères (non exhaustifs) du tableau 1 qui déterminent que l'ancien haut allemand et l'ancien néerlandais ont beaucoup de traits de langues syllabiques

\begin{tabular}{|l|l|l|}
\hline $\mathrm{n}^{\mathbf{0}}$ & critère & raison \\
\hline 1 & structure syllabique & $\begin{array}{l}\text { beaucoup moins complexes qu'en haut allemand moderne et en } \\
\text { néerlandais moderne, beaucoup de syllabes ouvertes }\end{array}$ \\
\hline 2 & frontières syllabiques & nettes \\
\hline 3 & géminées & existent en position héterosyllabique \\
\hline 6 & phonotactique & pas encore de dévoisement final en haut allemand \\
\hline 7 & vocalisme & $\begin{array}{l}\text { pas de réduction de voyelle en ancien haut allemand et en ancien } \\
\text { néerlandais }\end{array}$ \\
\hline 8 & $\begin{array}{l}\text { harmonie vocalique, } \\
\text { métaphonie }\end{array}$ & productive en ancien haut allemand \\
\hline
\end{tabular}

Nous pouvons donc constater que tandis que l'ancien haut allemand et l'ancien néerlandais étaient encore des langues syllabiques ${ }^{18}$, le haut allemand moderne et le néerlandais moderne sont très nettement des langues de mots. Leur évolution est donc l'inverse de celle du français.

\section{Conclusion}

Dans cette communication, j'ai montré que l'accent d'intensité fort du gallo-roman ne peut avoir de source francique, parce qu'autour du Ve siècle, date du prétendu emprunt de l'intensité, le francique ne peut avoir eu d'accent d'intensité fort et que la règle d'accentuation de l'ancien francique (avec l'accent sur la première syllabe de la racine) n'a pas été empruntée. ${ }^{19}$ J'ai montré également qu'a partir d'environ le XIIe siècle, le français et le francique se sont développés dans des directions opposées : alors que le français est devenu beaucoup plus une langue syllabique (selon la typologie d'Auer et d'Uhmann), le haut allemand et le néerlandais, les héréditaires du francique, sont devenus des langues de mots. 
Probablement, les auteurs des manuels qui attribuent l'accent d'intensité fort du gallo-roman à une influence francique ne se sont pas rendus compte du fait que le francique, tout comme le français, a connu de grandes évolutions typologiques entre le Ve et le XXe siècles, et ont projeté l'état du haut allemand et du néerlandais du XXe siècle (c.-à-d. des langues de mots avec un accent d'intensité fort) sur celui du francique du Ve siècle.

Cette communication montre, j'espère, que l'approche typologique en linguistique historique peut apporter ses fruits pour la compréhension des changements d'organisation phonologique dans une langue.

\section{Références bibliographiques}

Abercrombie, D. (1967). Elements of General Phonetics. Edimbourg : Edinburgh University Press.

Appel, R. et P. Muysken (1987). Language Contact and Bilingualism. Londres : Edward Arnold.

Auer, P. (1993). Is a rhythm-based typology possible? Universität Konstanz. Téléchargeable de : http://www.germanistik.uni-freiburg.de/auer/?Personal: Prof._Dr._Peter_Auer:Publikationen\#Online.

Auer, P. (1994). Einige Argumente gegen die Silbe als universale prosodische Hauptkategorie. Dans : Ramers, K.-H. et al. (éds.), Universale phonologische Strukturen und Prozesse. Tübingen : Niemeyer, 55-78.

Auer, P. (2001). Silben- und akzentzählende Sprachen. Dans: Haspelmath, Martin, Ekkehard König, Wulf Oesterreicher \& Wolfgang Raible (éds.). Language Typology and Language Universals. An International Handbook. Berlin : de Gruyter, 1391-1399.

Auer, P. \& S. Uhmann. (1988). Silben- und Akzentzählende Sprachen. Zeitschrift für Sprachwissenschaft 7/2, 21459.

Booij, G. (1995). The Phonology of Dutch. Oxford : Oxford University Press.

Braune, W. (1975). Althochdeutsche Grammatik. 13. Auflage. Bearbeitet von Hans Eggers. Tübingen : Niemeyer.

Braune, W. (1994). Althochdeutsches Lesebuch. 17. Auflage. Tübingen : Niemeyer.

Brüch, J. (1913). Der Einfluss der germanischen Sprachen auf das Vulgärlatein. Heidelberg : Carl Winter.

Cohen, M. (1967). Histoire d'une langue : le français. $3^{\mathrm{e}}$ édition. Paris : Editions sociales.

Cowan, H.K.J. (1957). De Oudnederlandse (Oudnederfrankische) psalmenfragmenten. Leiden : Brill.

D'Alquen, R. (1988). Germanic accent, grammatical change and the laws of unaccented syllables. New York: Lang.

Dauer, R.. (1983). Stress and syllable-timing reanalysed. Journal of Phonetics 11, 51-62.

Dauer, R. (1987). "Phonetic and phonological components of language rhythm". Proceedings of the XIth International Congress of Phonetic Sciences. Vol. 5. Tallinn: Academy of Sciences of the Estonian S.S.R., 447450.

Fournier, J.-M. (2007). From a Latin syllable-driven stress system to a Romance versus Germanic morphology-driven dynamics: in honour of Lionel Guierre. Language Sciences 29, 218-236.

Frei, E. (1988). Wortteilung und Silbenstruktur im Althochdeutschen. Munich : CD Copy und Druck.

Good, J. (à paraître). A twice-mixed creole? Tracing the history of a prosodic split in the Saramaccan lexicon. Studies in Linguistics. Téléchargeable de : http://www.acsu.buffalo.edu/ jcgood/jcgood-Saramaccan-Mixed.pdf.

Jacobs, H., 1992. The interaction between the evolution of syllable structure and foot structure in the historical phonology of French. Dans: Terrel, Morgan \& Christiane Laueffer (éds.), Theoretical Analyses in Romance Linguistics. Amsterdam : John Benjamins, 55-79.

König, W. (1978). DTV-Atlas zur deutschen Sprache. Munich : Deutscher Taschenbuch Verlag.

Kukenheim, L. (1971). Rôle de la prosodie dans l'histoire de la langue française. Dans : Cluzel, I. \& F. Pirot (éds.) Mélanges de Philologie romane dédiés à Jean Boutière, vol 1. Liège : Soledi, 317-331.

Kyes, R.L. (1969). The Old Low Franconian Psalms and Glosses. Ann Harbor, Mich. : University of Michigan Press. 
La Chaussée, F. de. (1989). Initation à la phonétique de l'ancien français. Nouvelle édition revue et augmentée. Paris : Klincksieck.

Nespor, M. et I. Vogel (1986). Prosodic Phonology. Dordrecht : Foris Publications.

Nübling, D. \& R. Schrambke. (2004). "Silben- versus akzentsprachliche Züge in germanischen Sprachen und im Alemannischen". Dans: Glaser, Elvira, Peter Ott, \& Rudolf Schwarzenbach (éds.), Alemannisch im Sprachvergleich. Beiträge zur 14. Arbeitstagung für alemannische Dialektologie in Männedorf (Zürich) vom 16.18.9.2002. Stuttgart : Franz Steiner, 280-320.

Pike, K. (1945). The Intonation of American English. Ann Arbor : University of Michigan Press.

Pope, M. (1952). From Latin to Modern French. 2ème édition. Manchester: Manchester University Press.

Prokosch, E. (1939). A comparative Germanic Grammar. Philadelphia, Penn.: University of Philadelphia Press \& Linguistic Society of America.

Roach, P. (1982). On the Distinction between "Stress-Timed" and "Syllable-Timed" Languages. Dans Cristal, D. (éd.), Linguistic Controversies. Essays in Linguistic Theory and Practice in Honour of F.R. Palmer. Londres : Edward Arnold, 73-79.

Quak, A. (1997). Oudnederlands. Dans : Van den Toorn, M.C., W. Pijnenburg, J.A. van Leuvensteijn et J.M. van der Horst (éds.), Geschiedenis van de Nederlandse taal. Amsterdam : Amsterdam University Press.

Richter, E. (1934). Beiträge zur Geschichte der Romanischen. I. Chronologische Phonetik des französischen bis zum Ende des 8. Jahrhunderts. Halle (Saale) : Niemeyer.

Schönfeld, M. (1934). Een Oudnederlandsche zin uit de elfde eeuw (met reproduktie). Tijdschrift voor Nederlandsche Taal en Letterkunde 52, 1-8.

Szczepaniak, R. (2007). Der phonologisch-typologische Wandel des Deutschen von einer Silben- zu einer Wortsprache. Berlin : de Gruyter.

Thomason, S.G. (2001). Language Contact. Edimbourg : Edinburgh University Press.

Verluyten, S.P. (1988). Introduction. Dans Verluyten, S.P. (éd.), La phonologie du schwa français. Amsterdam: Benjamins.

Van Helten, W.L. (1902). Die altostniederfränkischen Psalmenfragmente, die Lipsiusschen Glossen und die altsüdmittelfraenkischen Psalmenfragmente. Groningue : Wolters.

Von Wartburg, W. (1965). Evolution et structure de la langue française. $7^{\mathrm{e}}$ édition révisée. Berne : Francke.

Weinrich, U. (1963). Languages in Contact. La Haye : Mouton.

Wenk, B. \& F. Wioland (1982). Is French really syllable-timed? Journal of Phonetics 10, 193-216.

Werner, O. (1978). Schwa-Schwund und Phonotaktik im Deutschen. Dans Birnbaum, Henrik et al. (éds.), Studia Linguistica Alexandro Vasilii filio Issatschenko a Collegis Amicisque oblata. Lisse : The Peter de Ridder Press, 471-486.

Winford, D. (2003). An Introduction to Contact Linguistics. Malden, Mass. : Blackwell

Zink, G. (1986). Phonétique historique du français. Paris : Presses Universitaires de France.

\footnotetext{
* Je voudrais remercier Wolfgang Kehrein, Robert Kyes, Stephen Laker, Pieter Muysken, Arend Quak, Norval Smith, Marleen Van Peteghem, Piet van Reenen et un rapporteur anonyme de leur aide durant la préparation de cet article. Toutes les erreurs restantes sont les miennes.

${ }^{1}$ L'idée d'une influence du superstrat francique sur l'accentuation n'a pas été acceptée sans réserve par tous les linguistes (malgré le fait que l'on la retrouve abondamment dans les manuels). Par exemple, Cohen (1967: 109) exprime quelques doutes: "Les grands effets de l'accentuation (disparition des voyelles après l'accent) ont quelquefois été attribués à l'influence germanique, plus particulièrement francique, bas-allemande. De même certaines diphtongues. Mais l'ensemble des altérations n'est pas de type germanique. »
} 


\begin{abstract}
${ }^{2}$ L'allemand et le néerlandais fournissent des exemples de morphèmes empruntés qui ont gardé leur accentuation, par exemple les suffixes verbaux -ier/-eer, les suffixes nominaux -ität/-iteit, et les suffixes adjectivaux -el/-eel qui ont été empruntés au français et qui attirent l'accent. De même, le samaraccan (langue créole du Surinam) a un système prosodique 'fendu' : un type de prosodie (accentuation) 'européen' vient de l'anglais et du portugais et s'applique à des éléments d'origine anglaise et portugaise dans la langue, tandis que l'autre type de prosodie (système de tons) vient de langues africaines et s'applique aux éléments africains dans la langue (Good, à paraître). Mais dans les deux cas, celui de l'allemand et celui du samaraccan, l'accentuation reste liés aux mots ou aux morphèmes d'origine et ne se propage pas dans le reste de la langue.
\end{abstract}

${ }^{3}$ Dans cette sous-section et la suivante, il sera question de l'ancien francique du Ve au XIe siècle. Le lecteur ayant quelques connaissances de la linguistique historique du germanique remarquera peut-être qu'au cours de cette période, il y a eu une bifurcation en germanique occidental entre les dialectes qui ont subi la seconde mutation consonantique et ceux qui ne l'ont pas subie. Cette division a touché entre autres le francique, qui s'est divisé en bas francique et en haut francique (de ces deux dialectes, parmi d'autres dialectes, sont issus, avec respectivement le néerlandais et le haut allemand). Cependant cette division n'a pas de pertinence par rapport aux arguments qui vont être avancés ici, parce que les faits dont il sera question plus bas sont les mêmes pour ces deux variétés du germanique occidental.

${ }^{4}$ Il s'agit d'un nombre de mots isolés recueillis dans les Glosses de Malberg, ainsi que la phrase : maltho thi afrio lito 'je te dis : je te libère, serf'. La différence avec le néerlandais et le haut-allemand d'aujourd'hui est très considérable et il est difficile d'y reconnaître une langue germanique.

${ }^{5}$ Il existe cependant des mots d'emprunt d'origine germanique en latin vulgaire, cf. Brüch (1913).

${ }^{6}$ La loi de Verner a fonctionné dans les Ier et IIe siècles en germanique ; ses effets ont été rendus phonémiques par un glissement de l'accent allant d'une place libre en indo-européen vers la première syllabe de la racine. Ce glissement a donc opéré après le fonctionnement de la loi de Verner, mais a été complété avant le Ve siècle (Prokosch 1939 : 62-64, D’Alquen 1988).

${ }^{7}$ On trouve un exemple de réduction partielle en russe, où le contraste entre /a/ et /o/ ainsi que celui entre /i/ et /e/ est neutralisé en position inaccentuée.

${ }^{8}$ Le $n$ à la fin de himeln (au lieu du $m$ dans l'exemple de l'ancien haut francique) s'explique par le fait qu'ici, le mot est au pluriel ainsi que par la présence de l'article.

${ }^{9}$ Un rapporteur anonyme a remarqué que la datation de Richter n'est pas acceptée par tous. Cependant, il est certain que ces changements se sont produits avant le milieu du IX siècle, donc largement antérieur à l'avènement de la réduction de voyelle en francique.

${ }^{10}$ D'Alquen $(1988: 17,232)$ conclue qu'à l'époque du fonctionnement de la loi de Verner (Ie et IIe siècles), l'accent germanique était mélodique. Il n'y a aucune indication qu'au Ve siècle, il serait devenu tout d'un coup fortement expiratoire.

${ }^{11}$ En général dans les langues romanes, il y a eu une évolution de l'accent mélodique du latin classique vers un accent d'intensité (Cohen 1967 : 50). Pour le latin en Gaule, ce développement s'est amorcé au plus tard au IVe siècle (Pope 1952 : 99), donc avant l'invasion des Francs en Gaule. Cependant, en gallo-roman l'intensification de l'accent est allée plus loin que dans les autres langues romanes. On sait d'ailleurs de la phonétique expérimentale que l'accent peut avoir son expression en hauteur (fréquence fondamentale), en intensité et en durée. La plupart du temps, pour la réalisation de l'accent, il s'agit d'un mélange de ces trois dimensions.

${ }^{12}$ L'effacement du schwa en français moderne a fait l'objet d'une vaste littérature en phonologie moderne. D'autres facteurs que le conditionnement syllabique sont présentés dans cette littérature, notamment des facteurs rythmiques et morphologiques cf. Verluyten (1988: 4-10).

${ }^{13}$ Pour le haut allemand, ce développement a été progressif pendant plusieurs siècles successifs, à partir du moyen haut allemand (Werner 1978). Ce développement a été appelé Konsonantenhäufung 'entassement de consonnes' par les historiens du haut allemand.

${ }^{14}$ Selon Quak (1997) et d'autres descriptions du moyen néerlandais, la genèse du dévoisement final en néerlandais aurait devancé celle du haut allemand. 
15 'Prosodique' signifie ici : 'ayant trait aux constituantes prosodiques', et non pas : 'ayant trait à la mélodie de phrase.'

${ }^{16}$ Ce tableau a été adapté de Nübling \& Schrambke (2004 : 284-285). AHA = ancien haut allemand, NHA = nouveau haut allemand.

${ }^{17}$ Cette liste a été dressée originalement pour montrer des différences typologiques sur l'échelle : langues de mots langue syllabiques, entre des langues et dialectes germaniques tels que le haut allemand, le néerlandais, le danois et l'anglais d'une part (langue de mots) et des dialectes alémaniques méridionales, l'afrikaans, le suédois et le norvégien de l'autre (langues syllabiques). Le luxembourgeois se trouverait dans le milieu de l'échelle.

${ }^{18}$ Nübling \& Schrampke (2004 : 290) réfèrent à Frey (1988), qui fournit des arguments pour lesquels l'ancien haut allemand doit être catégorisé comme étant beaucoup une langue syllabique ('... wobei das Althochdeutsche noch stark als silbensprachlich einzustufen ist').

${ }^{19}$ Comme l'a remarqué un rapporteur anonyme, cela va de pair avec la constatation que l'on trouve des phénomènes de syncope et d'apocope également dans d'autres langues romanes, où il n'y a pas d'influence francique, comme le catalan et le castillan. 